\title{
STEADY STATE AND SCALING LIMIT FOR A TRAFFIC CONGESTION MODEL
}

\author{
ILIE GRIGORESCU ${ }^{1}$ AND Min KANG ${ }^{2}$
}

\begin{abstract}
In a general model (AIMD) of transmission control protocol (TCP) used in internet traffic congestion management, the time dependent data flow vector $\mathbf{x}(t)>0$ undergoes a biased random walk on two distinct scales. The amount of data of each component $x_{i}(t)$ goes up to $x_{i}(t)+a$ with probability $1-\zeta_{i}(\mathbf{x})$ on a unit scale or down to $\gamma x_{i}(t), 0<\gamma<1$ with probability $\zeta_{i}(\mathbf{x})$ on a logarithmic scale, where $\zeta_{i}$ depends on the joint state of the system $\mathbf{x}$. We investigate the long time behavior, mean

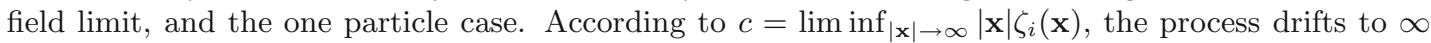
in the subcritical $c<c_{+}(n, \gamma)$ case and has an invariant probability measure in the supercritical case $c>c_{+}(n, \gamma)$. Additionally, a scaling limit is proved when $\zeta_{i}(\mathbf{x})$ and $a$ are of order $N^{-1}$ and $t \rightarrow N t$, in the form of a continuum model with jump rate $\alpha(\mathbf{x})$.
\end{abstract}

Mathematics Subject Classification. 60K30, 60J25, 90B20.

Received September 28, 2007. Revised June 27, 2008.

\section{InTRODUCTION}

In a general model used in internet congestion control $[2,9,10,14,17,19]$, related to classical autoregressive models [7,18], the time dependent data flow $x(t)$ undergoes a biased random walk with linear steps in one direction ( $x$ moves to $x+a, a>0)$ and on a logarithmic scale in the other $(x$ moves to $\gamma x$, where $0<\gamma<1$ ), belonging to a class of dynamics known in the literature [14] as AIMD (additive increase multiplicative decrease). In the present paper, we concentrate on one standard model, defined rigorously as the solution to the martingale problem given by (2.3), which we shall call the $\gamma$-process.

More precisely, assume $(\Omega, \Sigma, P)$ is a probability space, $\left\{\mathcal{F}_{t}\right\}_{t \geq 0}$ is a filtration on $(\Omega, \Sigma)$. Let $n$ be a positive integer and $\zeta_{i}:[0, \infty) \times(0, \infty)^{n} \rightarrow[0,1], 1 \leq i \leq n$ be continuous functions. In addition, we consider a family of $n$ independent Poisson processes $\left\{\pi_{i}(t)\right\}_{1 \leq i \leq n}$ with rate $\lambda>0$, adapted to the filtration $\left\{\mathcal{F}_{t}\right\}_{t \geq 0}$.

For any starting point $\mathbf{x}_{0}$ with components $x_{0 i}, 1 \leq i \leq n$, let $\mathbf{x}(t)$ denote the pure jump Markov process on $(0, \infty)^{n}$ with components $\left(x_{1}(t), x_{2}(t), \ldots, x_{n}(t)\right)$, constructed as follows. A Poisson clock $\pi_{i}(t)$ with intensity $\lambda>0$ is attached to each particle $x_{i}(t), 1 \leq i \leq n$, with all clocks mutually independent. When the Poisson clock $\pi_{i}$, associated to the particle $x_{i}(t)$, rings at time $\tau, x_{i}$ moves to $x_{i}(\tau-)+a$ with probability $1-\zeta_{i}(\tau, \mathbf{x}(\tau-))$

\footnotetext{
Keywords and phrases. TCP, AIMD, fluid limit, mean field interaction.

1 Department of Mathematics, University of Miami, Coral Gables, FL 33124-4250, USA; igrigore@math.miami.edu

2 Department of Mathematics, North Carolina State University, Raleigh, NC 27695, USA; kang@math.ncsu.edu
} 
and to $\gamma x_{i}(\tau-)$ with probability $\zeta_{i}(\tau, \mathbf{x}(\tau-))$. In this standard construction of a pure jump process, there are no simultaneous jumps.

This is a multiple particle process $\mathbf{x}(t)$, to be introduced rigorously in Section 2, where particles $x_{i}$ (or different server rates) interact through their jump probabilities $\zeta_{i}(t, \mathbf{x})$. It can be regarded as a generalization of a continuous time linear state space model LSS(F, G) (page 9 in [18]) with driving matrices $F$ and $G$ depending on the trajectory of the process. The only case when the autoregressive models (see extensive references in [7]) coincide with a skeleton of the Markovian process given here is when the jump rates $\zeta_{i}$ are constant.

We are exploring the model in the following directions:

1) Theorem 2.1 in Section 2 gives sufficient bounds on the rates $\zeta_{i}$ assuring the existence of an invariant probability measure of the system while not assuming any particular nature of the interaction (mean-field or otherwise), of the form $\zeta_{i}(t, \mathbf{x}) \sim c /|\mathbf{x}|$ when $\mathbf{x}$ is large and $\zeta_{i}(t, \mathbf{x}) \sim O(1)$ (bounded away from zero) when $\mathbf{x}$ is small. The theorem provides sharp bounds (see the discussion thereafter). Most importantly, it establishes a

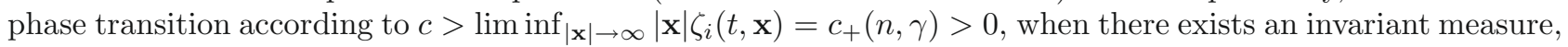
and $c<c_{+}(n, \gamma)$, when the particles drift to infinity in mean value. As different orders of magnitude lead to transient behavior of the process (see remarks after the theorem), the bounds are optimal.

Various orders of magnitude of the jump size are considered in recent papers $[14,17,19]$ under constant $\zeta$ but this is the first instance to our knowledge where the rates themselves are analyzed and the natural scale and criticality are identified. This approach is particularly important and the natural one since in the main application of the model, in which $\zeta$ represents the probability of loss of packets of information $\mathbf{x}$, one expects non-increasing behavior in each component of $\mathbf{x}$ (or, in other models, the "average" level of congestion $\bar{x}$ ). In this case the assumptions $(C+)$ and $(C-)$ of Theorem 2.1 are immediately satisfied with the exception of the trivial case $\zeta \equiv 0$.

It is useful to note in the discrete setting, where $a=1$, the left side jump is $x_{i} \rightarrow\left[\gamma x_{i}\right]$ and a lower bound away from zero on $x$ is imposed, the irreducibility of the process is immediate and Theorem 2.1 implies the existence of an unique invariant measure - see [19] for related models.

2) The mean-field case from Section 3 appears in the engineering literature $[1,5,6,9,10]$, studied via simulations in $[1,6]$ and considered in $[2,3]$ with a related but different model. Here $\zeta_{i}(t, \mathbf{x})=p(t, \bar{x})$ depends on $t$ and the average $\bar{x}=n^{-1} \sum x_{i}$. This arises as the simplest interactive setup in which the data flows $x_{i}$ depend on the total transmission rate. Mean field traffic control protocols may be used when a large number of individual flows are present. In case of large data loads, it would be ideal to identify the main individual contributors and control those specific flows with large loads. However, in reality, it may be difficult to identify those main contributors and an "average"-dependent protocol is appropriate. One could consider the representation of the effects of TCP on a large space-time scale as a processor sharing queue where every flow receives the same share of the total capacity of the link. Then, under homogenization as in Theorem 3.1, the bulk behavior can be analyzed via a differential equation (3.8). Mathematically, the mean field model is important because it is closable: as $n \rightarrow \infty$, the differential equation (3.9) governing the limit of the empirical process (3.1) emerges in explicit, closed form. Section 4 looks at the one particle process $(n=1)$. Proposition 4.1 establishes the uniqueness of the solution to the fluid limit equation (3.1) and Theorem 4.1 gives the explicit form of the invariant measure in the simplest case $(n=1, \zeta=$ const.).

3) Section 5 pursues the multi-particle case $(n=N)$ when $p(t, N x) \simeq N^{-1} \alpha(t, x)$ under time speed up $t \rightarrow N t$ for a large scaling factor $N$ (consistent with the analysis in Thm. 2.1), proving a full hydrodynamic limit for the empirical measure (5.1) in Theorem 5.1. The result relates partially to the limiting process from equation (2.1) in [19] ( $\alpha=$ constant, $n=1$, non-interactive dynamics) and the convergence to the process given by equation (2.7) quoted in [14] from [8] $(\alpha(x)=x, n=1$, non-interactive dynamics). Recurrence issues and the ergodic properties of the one-particle scaling limit process with rate $\alpha(x)$ are analyzed in greater detail in $[13]$. 


\section{THE GENERAL MODEL}

\subsection{Martingale problem and a class of test functions}

Due to the natural bounds $x_{0 i} \gamma^{\pi_{i}(t)} \leq x_{i}(t) \leq x_{0 i}+a \pi_{i}(t), 1 \leq i \leq n$ and $t \geq 0$,

$$
E\left[\exp \left(\eta x_{i}(t)\right)\right] \leq \exp \left(\eta x_{0 i}+\lambda t\left(\mathrm{e}^{a \eta}-1\right)\right), \quad 0 \leq \eta<\infty
$$

additionally, all negative moments are finite and

$$
E\left[\left(x_{i}(t)\right)^{m}\right] \leq x_{0 i}^{m} \mathrm{e}^{\lambda t\left(\gamma^{m}-1\right)}, \quad-\infty<m \leq 0
$$

The process $\{\mathbf{x}(t)\}$ can be seen as the solution to a martingale problem. Denote $R_{i} \mathbf{x}=\left(x_{1}, x_{2}, \ldots, x_{i}+a, \ldots, x_{n}\right)$ and $L_{i} \mathbf{x}=\left(x_{1}, x_{2}, \ldots, \gamma x_{i}, \ldots, x_{n}\right)$. For a test function $f \in C_{b}\left([0, \infty) \times(0, \infty)^{n}\right)$ - the space of continuous bounded functions in $(t, \mathbf{x})$ - let $\mathcal{M}_{f}(t)$ be defined by the stochastic differential formula

$$
\begin{aligned}
\mathcal{M}_{f}(t)=f(t, \mathbf{x}(t))-f(0, \mathbf{x}(0))-\int_{0}^{t}\left\{\partial_{s} f(s, \mathbf{x}(s-))\right. & +\lambda \sum_{i=1}^{n}\left[\left(1-\zeta_{i}(s, \mathbf{x}(s-))\right)\left(f\left(s, R_{i} \mathbf{x}(s-)\right)-f(s, \mathbf{x}(s-))\right)\right. \\
& \left.\left.+\zeta_{i}(s, \mathbf{x}(s-))\left(f\left(s, L_{i} \mathbf{x}(s-)\right)-f(s, \mathbf{x}(s-))\right)\right]\right\} \mathrm{d} s . \quad(2.3)
\end{aligned}
$$

Then, $\mathcal{M}_{f}(t)$ is a martingale with quadratic variation

$$
\begin{aligned}
\left\langle\mathcal{M}_{f}\right\rangle(t)= & \lambda \int_{0}^{t} \sum_{i=1}^{n}\left[\left(1-\zeta_{i}(s, \mathbf{x}(s-))\right)\left(f\left(s, R_{i} \mathbf{x}(s-)\right)-f(s, \mathbf{x}(s-))\right)^{2}\right. \\
& \left.+\zeta_{i}(s, \mathbf{x}(s-))\left(f\left(s, L_{i} \mathbf{x}(s-)\right)-f(s, \mathbf{x}(s-))\right)^{2}\right] \mathrm{d} s .
\end{aligned}
$$

The martingale problem (2.3)-(2.4) can be formulated with functions in a larger space, including exponentials and, in particular, polynomial functions, that will be used repeatedly.

Definition 2.1. Given $\eta>0$, let $\phi \in C_{\eta}^{1,2}\left([0, \infty) \times(0, \infty)^{n}\right)$ be functions in $C^{1,2}\left([0, \infty) \times(0, \infty)^{n}\right)$ with partial derivatives $\partial_{t}^{a} \partial_{\mathbf{x}}^{\mathbf{b}} \phi, 0 \leq a \leq 1,0 \leq|\mathbf{b}| \leq 2$ having exponential moments up to $\eta$ as $|\mathbf{x}| \rightarrow \infty$ and negative moments as $|\mathbf{x}| \rightarrow 0$. More precisely, there exists $k>0$ and a positive constant $K_{\phi}(k)$ such that

$$
\sup _{0 \leq a \leq 1,0 \leq|b| \leq 2} \sup _{(t, \mathbf{x}) \in[0, \infty) \times(0, \infty)^{n}}|\mathbf{x}|^{k} \mathrm{e}^{-\eta|\mathbf{x}|}\left|\partial_{t}^{a} \partial_{\mathbf{x}}^{b} \phi(t, \mathbf{x})\right|=K_{\phi}(k)<\infty .
$$

Here $\mathbf{b}=\left(b_{1}, \ldots, b_{n}\right)$ is a multi-index with nonnegative integer entries and $|\mathbf{b}|=\sum_{j=1}^{n} b_{j}$. The notation stands for $\partial_{t}^{a} \partial_{\mathbf{x}}^{\mathbf{b}}=\frac{\partial^{a} \partial^{b_{1} \ldots .} \partial^{b_{n}}}{\partial t^{a} \partial x_{1}^{b_{1}} \ldots \partial x_{n}^{b_{n}}}$ and $a=0, b_{j}=0$ means no derivative is taken.

Remark. Power functions and exponential functions obviously belong to $C_{\eta}^{1,2}\left([0, \infty) \times(0, \infty)^{n}\right)$ for some $\eta>0$. Due to the integrability conditions (2.1) and (2.2), the stochastic differential formulas (2.3)-(2.4) can be extended to test functions in the class $C_{\eta}^{1,2}\left([0, \infty) \times(0, \infty)^{n}\right)$.

\subsection{Existence of invariant measures}

A Poisson process escapes towards infinity as $t \rightarrow \infty$. However, lower and upper bounds on the rate $\zeta_{i}$ of moving backwards $x \rightarrow \gamma x$ ensure that the process has at least one invariant probability measure on the interval $(0, \infty)$. In the following theorem, we shall take $a=1, \lambda=1$ without any loss of generality. 
Theorem 2.1. Let $c_{+}(n, \gamma)=\frac{n}{1-\gamma}$. Assume that, uniformly in $t \geq 0$, the jump rates satisfy

$(C+)$ for all $i, 1 \leq i \leq n, \liminf _{|\mathbf{x}| \rightarrow \infty}|\mathbf{x}| \zeta_{i}(t, \mathbf{x})>c_{+}(n, \gamma)$, and

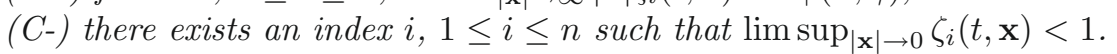

Then, the process defined by (2.3)-(2.4) has tight average occupation measures (2.8). In the time-homogeneous case the process has at least one invariant probability measure concentrated on $(0, \infty)$.

Remark 2.1. A simple sufficient condition for $(\mathrm{C}+)$ is $\liminf _{|\mathbf{x}| \rightarrow \infty}|\mathbf{x}|^{b} \zeta_{i}(t, \mathbf{x})>0$ for some $b \in[0,1)$. The bound $c_{+}(n, \gamma)$ is nontrivial when $b=1$, and reflects the critical nature of $\zeta_{i}(t, \mathbf{x}) \sim O\left(|\mathbf{x}|^{-1}\right)$, present in the scaled model from Section 5 , where $\zeta(t, N \mathbf{x}) \simeq$ const. $N^{-1}$. More precisely, $t^{-1} \int_{0}^{t} E|\mathbf{x}(s)| \mathrm{d} s \sim O(1)$ when $c>c_{+}(n, \gamma)$ and $\sim O(t)$ (transient case) when $c<c_{+}(n, \gamma)$. For example, take $n=1$ and $\zeta(t, x)=c(1+x)^{-1}$ for small $c$. Alternatively, if $\zeta(t, x)=(1+(1-\gamma) x)^{-1}$ the process $x(t)$ is a martingale. One can interpret $(C-)$ as a condition on the support of the invariant measure.

Remark 2.2. Conditions $(C+)$ and $(C-)$ do not guarantee the uniqueness of the invariant measure. Set $\zeta_{i} \equiv 0$ for some intermediate regime $\mathbf{x}$ in a finite box in $\mathbb{R}^{n}$ away from the origin. The state space breaks down into incommunicable classes and evidently there will be more than one invariant probability measures. One can strengthen the conditions with bounds over all $\mathbf{x}$, but this weakens the result; as mentioned earlier, see [13] for the detailed treatment of an AIMD model in one dimension (the proof in higher dimensions is essentially the same), including ergodicity on a general state space (continuum).

Remark 2.3. The two $m$ in Part $1(m>0)$ and Part $2(m<0)$ of the proof are obviously not the same (they have opposite signs). In Part 1 , the constant $c_{+}$must exceed $c_{+}(n, \gamma)$ which is achieved at $m=1$. On the other hand, for any $c_{-}>0$, there exists $m<0$ to satisfy $c_{-}>\gamma^{m}-1$, which makes $c_{-}$arbitrary; in other words $c_{-}(n, \gamma)=0$, by analogy with the notation for the upper bound $c_{+}(n, \gamma)$.

Proof. Condition $(\mathrm{C}+)$ implies that there exist $k_{+}>1, c_{+}>c_{+}(n, \gamma)$ such that, uniformly in $(t, \mathbf{x})$

$$
\zeta_{i}(t, \mathbf{x}) \geq c_{+}|\mathbf{x}|^{-1}, \quad|\mathbf{x}| \geq k_{+} .
$$

Similarly, for at least one index $i$, there exist $0<k_{-}<1, c_{-}>0$ such that, uniformly in $(t, \mathbf{x})$

$$
1-\zeta_{i}(t, \mathbf{x}) \geq c_{-}, \quad|\mathbf{x}| \leq k_{-} .
$$

Fix $\mathbf{x}_{0}=\left(x_{10}, \ldots, x_{n 0}\right) \in(0, \infty)^{n}$. Our goal is to prove the tightness of the family of probability measures on $(0, \infty)^{n}$

$$
\nu_{t}(\mathrm{~d} \mathbf{x})=t^{-1} \int_{0}^{t} P_{\mathbf{x}_{0}}(s, \mathrm{~d} \mathbf{x}) \mathrm{d} s, \quad t>0
$$

where $P_{\mathbf{x}_{0}}(s, \mathrm{~d} \mathbf{x})=P\left(\mathbf{x}(s) \in \mathrm{d} \mathbf{x} \mid \mathbf{x}(0)=\mathbf{x}_{0}\right)$.

The family of probability measures $\left\{\nu_{t}\right\}_{t>0}$ is tight if there exists $M>1$ such that

$$
\lim _{M \rightarrow \infty} \limsup _{t \rightarrow \infty} \nu_{t}\left(\left\{M^{-1} \leq|\mathbf{x}| \leq M\right\}^{c}\right)=0 .
$$

We shall break down the proof into two parts, showing respectively

$$
\lim _{M \rightarrow \infty} \limsup _{t \rightarrow \infty} \nu_{t}(\{|\mathbf{x}|>M\})=0, \quad \lim _{M \rightarrow \infty} \limsup _{t \rightarrow \infty} \nu_{t}\left(\left\{|\mathbf{x}|<M^{-1}\right\}\right)=0 .
$$

We shall denote by $d_{l}$ and $D_{l}$ the positive constants such that for all $\mathbf{x} \in \mathbb{R}^{n}$

$$
d_{l}|\mathbf{x}|^{l} \leq \sum_{i=1}^{n} x_{i}^{l} \leq D_{l}|\mathbf{x}|^{l}, \quad l>0
$$


The moments $E\left[x_{i}^{m}(t)\right], 1 \leq i \leq n$ are finite for both $m$ positive and negative due to the bounds (2.1)-(2.2) and the stochastic differential formulas (2.3)-(2.4) are valid for power functions as mentioned in the remark following Definition 2.1.

We apply the expected value in (2.3) to the function $f(\mathbf{x})=\sum_{i=1}^{n} x_{i}^{m}$ on a time interval $0 \leq s \leq t$ to obtain

$$
\begin{aligned}
E\left[\sum_{i=1}^{n} x_{i}^{m}(t)\right]= & \sum_{i=1}^{n} x_{i}^{m}(0) \\
& +\int_{0}^{t} E\left[\sum _ { i = 1 } ^ { n } \left\{\left(1-\zeta_{i}(s, \mathbf{x}(s-))\right)\left[\left(x_{i}(s-)+1\right)^{m}-x_{i}^{m}(s-)\right]\right.\right. \\
& \left.\left.-\left(1-\gamma^{m}\right) \zeta_{i}(s, \mathbf{x}(s-)) x_{i}^{m}(s-)\right\}\right] \mathrm{d} s
\end{aligned}
$$

Part 1. The upper bound in (2.10). Since $c_{+}(n, \gamma)=\lim _{m \downarrow 1} \frac{n m 2^{m-1}}{d_{m}\left(1-\gamma^{m}\right)}$, whenever (2.6) holds, there exists $m>1$ satisfying $c_{+}>\frac{n m 2^{m-1}}{d_{m}\left(1-\gamma^{m}\right)}$. Using the bounds $\zeta_{i}(s, \mathbf{x}(s-)) \geq c_{+}|\mathbf{x}(s-)|^{-1}$ as well as the trivial bound $1-\zeta_{i}(s, \mathbf{x}(s-)) \leq 1$, we have an upper bound

$$
E\left[\sum_{i=1}^{n} x_{i}^{m}(t)\right] \leq \sum_{i=1}^{n} x_{i}^{m}(0)+I_{1}+I_{2}
$$

where

$$
I_{1}=\int_{0}^{t} E\left[\left(\sum_{i=1}^{n}\left[m\left(x_{i}(s-)+1\right)^{m-1}-\left(1-\gamma^{m}\right) c_{+} \frac{x_{i}^{m}(s-)}{|\mathbf{x}(s-)|}\right]\right) \mathbf{1}_{\left[k_{+}, \infty\right)}(|\mathbf{x}(s-)|)\right] \mathrm{d} s
$$

and

$$
I_{2}=\int_{0}^{t} E\left[\left(\sum_{i=1}^{n}\left[m\left(x_{i}(s-)+1\right)^{m-1}\right]\right) \mathbf{1}_{\left(0, k_{+}\right)}(|\mathbf{x}(s-)|)\right] \mathrm{d} s \leq n m\left(1+k_{+}\right)^{m-1} t .
$$

The terms $\left(x_{i}(s-)+1\right)^{m-1}$ in integral $I_{1}$ are bounded above by $2^{m-1}|\mathbf{x}(s-)|^{m-1}$ and $\sum_{i=1}^{n} \frac{x_{i}^{m}(s-)}{|\mathbf{x}(s-)|} \geq d_{m}|\mathbf{x}(s-)|^{m-1}$ from (2.11). Re-writing the inequality after we move $I_{1}$ to the left hand side, we find

$$
\int_{0}^{t} E\left[|\mathbf{x}(s-)|^{m-1} \mathbf{1}_{\left[k_{+}, \infty\right)}(|\mathbf{x}(s-)|)\right] \mathrm{d} s \leq \frac{\sum_{i=1}^{n} x_{i 0}^{m}+n m\left(1+k_{+}\right)^{m-1} t}{d_{m} c_{+}\left(1-\gamma^{m}\right)-n m 2^{m-1}},
$$

where the coefficient $d_{m} c_{+}\left(1-\gamma^{m}\right)-n m 2^{m-1}$ from $I_{1}$ is positive by construction. Denote $C_{+}\left(t, \mathbf{x}_{0}\right)$ the right side of (2.14).

Let $M>0$ be a large number, $M \geq k_{+}+1$ without loss of generality. Since either $x_{i}(s)=x_{i}(s-)$, $x_{i}(s)=x_{i}(s-)+1$ or $x_{i}(s)=\gamma x_{i}(s-)$, then $x_{i}(s-) \geq x_{i}(s)-1$ and also $|\mathbf{x}(s-)| \geq|\mathbf{x}(s)|-1$. For $\nu_{t}$ defined in $(2.8)$,

$$
\nu_{t}(\{|\mathbf{x}|>M\})=\frac{1}{t} \int_{0}^{t} P_{\mathbf{x}_{0}}(|\mathbf{x}(s)|>M) \mathrm{d} s \leq \frac{\int_{0}^{t} E\left\{|\mathbf{x}(s-)|^{m-1} \mathbf{1}_{\left[k_{+}, \infty\right)}(|\mathbf{x}(s-)|)\right\} \mathrm{d} s}{t(M-1)^{m-1}}
$$

showing that

$$
\lim _{M \rightarrow \infty} \limsup _{t \rightarrow \infty} \nu_{t}\left(\{|\mathbf{x}| \leq M\}^{c}\right) \leq \lim _{M \rightarrow \infty} \limsup _{t \rightarrow \infty} \frac{C_{+}\left(t, \mathbf{x}_{0}\right)}{t(M-1)^{m-1}}=0
$$

which proves the upper bound for our claim. 
Part 2. The lower bound in (2.10). Without loss of generality we take $i=1$. Pick $m<0$ fixed but arbitrary and the test function $f(\mathbf{x})=\left(\sum_{i=1}^{n} x_{i}\right)^{m}$.

$$
E[f(\mathbf{x}(t))]=f(\mathbf{x}(0))+\sum_{i=1}^{n}\left(J_{11}^{i}+J_{12}^{i}+J_{21}^{i}+J_{22}^{i}\right)
$$

where

$$
\begin{gathered}
J_{11}^{i}=\int_{0}^{t} E\left\{\left[\left(1-\zeta_{i}(s, \mathbf{x}(s-))\right)\left[f\left(R_{i} \mathbf{x}(s-)\right)-f(\mathbf{x}(s-))\right]\right] \mathbf{1}_{\left(0, k_{-}\right]}(|\mathbf{x}(s-)|)\right\} \mathrm{d} s, \\
J_{12}^{i}=\int_{0}^{t} E\left\{\left[\zeta_{i}(s, \mathbf{x}(s-))\left[f\left(L_{i} \mathbf{x}(s-)\right)-f(\mathbf{x}(s-))\right]\right] \mathbf{1}_{\left(0, k_{-}\right]}(|\mathbf{x}(s-)|)\right\} \mathrm{d} s
\end{gathered}
$$

and

$$
\begin{gathered}
J_{21}^{i}=\int_{0}^{t} E\left\{\left[\left(1-\zeta_{i}(s, \mathbf{x}(s-))\right)\left[f\left(R_{i} \mathbf{x}(s-)\right)-f(\mathbf{x}(s-))\right]\right] \mathbf{1}_{\left(k_{-}, \infty\right)}(|\mathbf{x}(s-)|)\right\} \mathrm{d} s, \\
J_{22}^{i}=\int_{0}^{t} E\left\{\left[\zeta_{i}(s, \mathbf{x}(s-))\left[f\left(L_{i} \mathbf{x}(s-)\right)-f(\mathbf{x}(s-))\right]\right] \mathbf{1}_{\left(k_{-}, \infty\right)}(|\mathbf{x}(s-)|)\right\} \mathrm{d} s .
\end{gathered}
$$

We look for an upper bound for the right hand side. For all $i$, the terms $J_{11}^{i}$ and $J_{21}^{i}$ are less or equal to zero. All $J_{21}^{i}$ will be ignored, together with all $J_{11}^{i}$, except $J_{11}^{1}$ for $i=1$. In $J_{11}^{1}$ we have $\left(1-\zeta_{i}(s, \mathbf{x}(s-))\right) f\left(R_{i} \mathbf{x}(s-)\right) \leq 1$ and $-\left(1-\zeta_{i}(s, \mathbf{x}(s-))\right) f(\mathbf{x}(s-)) \leq-c_{-} f(\mathbf{x}(s-))$. In all $J_{12}^{i}, J_{22}^{i}$ we use the inequality $f\left(L_{i} \mathbf{x}(s-)\right)-f(\mathbf{x}(s-)) \leq$ $\left(\gamma^{m}-1\right) f(\mathbf{x}(s-))$. For $J_{22}^{i}$ we have the bound $f(\mathbf{x}(s-)) \leq d_{1}^{m} k_{-}^{m}$. We put together the upper bounds obtained for the terms multiplied by $\mathbf{1}_{\left(0, k_{-}\right]}(|\mathbf{x}(s-)|)$

$$
-\left(c_{-}-n\left(\gamma^{m}-1\right)\right) \int_{0}^{t} E\left\{f(\mathbf{x}(s-)) \mathbf{1}_{\left(0, k_{-}\right]}(|\mathbf{x}(s-)|)\right\} \mathrm{d} s+t
$$

and the upper bounds for the terms multiplied by $\mathbf{1}_{\left(k_{-}, \infty\right)}(|\mathbf{x}(s-)|)$, which are less or equal to $n\left(\gamma^{m}-1\right) d_{1}^{m} k_{-}^{m} t$. Based on (2.17), we move the term with factor $-\left(c_{-}-n\left(\gamma^{m}-1\right)\right)$ to the left hand side of the inequality and $E[f(\mathbf{x}(t))]$ to the right hand side; since the latter is nonnegative, it will be ignored. Choosing $m$ sufficiently close to zero, the factor $c_{-}-n\left(\gamma^{m}-1\right)$ becomes positive. We have proven the inequality

$$
\int_{0}^{t} E\left\{f(\mathbf{x}(s-)) \mathbf{1}_{\left(0, k_{-}\right]}(|\mathbf{x}(s-)|)\right\} \mathrm{d} s \leq \frac{f(\mathbf{x}(0))+\left[1+n\left(\gamma^{m}-1\right) d_{1}^{m} k_{-}^{m}\right] t}{c_{-}-n\left(\gamma^{m}-1\right)} .
$$

We denote $C_{-}\left(t, \mathbf{x}_{0}\right)$ the constant on the right hand side of $(2.22)$.

Let $M>0$ be a large number, $k_{-}>D_{1}\left(d_{1} \gamma M\right)^{-1}$ without loss of generality. Since either $x_{i}(s)=x_{i}(s-)$, $x_{i}(s)=x_{i}(s-)+1$ or $x_{i}(s)=\gamma x_{i}(s-)$, then $|\mathbf{x}(s)|<M^{-1}$ implies $|\mathbf{x}(s-)|<\gamma^{-1} M^{-1}$ and then $f(\mathbf{x}(s-))>$ $D_{1}^{m}(\gamma M)^{-m}$.

For $\nu_{t}$ defined in $(2.8)$,

$$
\begin{aligned}
& \nu_{t}\left(\left\{|\mathbf{x}|<M^{-1}\right\}\right)=\frac{1}{t} \int_{0}^{t} P_{\mathbf{x}_{0}}\left(|\mathbf{x}(s)|<M^{-1}\right) \mathrm{d} s \\
& \quad \leq \frac{1}{t} \int_{0}^{t} P_{\mathbf{x}_{0}}\left(f(\mathbf{x}(s-))>D_{1}^{m}(\gamma M)^{-m}\right) \mathrm{d} s \leq \frac{\int_{0}^{t} E\left\{f(\mathbf{x}(s-)) \mathbf{1}_{\left.\left(D_{1}^{m}(\gamma M)^{-m}\right), \infty\right)}(f(\mathbf{x}(s-)))\right\} \mathrm{d} s}{t D_{1}^{m}(\gamma M)^{-m}},
\end{aligned}
$$


using Chebyshev's inequality on the last line. The indicator function in the last integral is less than $\mathbf{1}_{\left(0, k_{-}\right]}$ $(|\mathbf{x}(s-)|)$ from $(2.22)$, showing that

$$
\lim _{M \rightarrow \infty} \limsup _{t \rightarrow \infty} \nu_{t}\left(\left\{|\mathbf{x}| \geq M^{-1}\right\}^{c}\right) \leq \lim _{M \rightarrow \infty} \limsup _{t \rightarrow \infty} \frac{C_{-}\left(t, \mathbf{x}_{0}\right)}{t D_{1}^{m} \gamma^{-m} M^{-m}}=0
$$

which proves the lower bound for our claim.

We put together (2.15)-(2.16) and (2.23)-(2.24), finishing the proof of (2.10).

\section{THE FLUID LIMIT FOR THE MEAN-FIELD MODEL}

Let $M_{1}(\mathbb{R})$ denote the space of probability measures on the set $\mathbb{R}$. We start the investigation by considering the empirical measure of the $n$ particle $\gamma$-process

$$
\mu^{n}(t, \mathrm{~d} x)=\frac{1}{n} \sum_{i=1}^{n} \delta_{x_{i}(t)}(\mathrm{d} x) \in M_{1}(\mathbb{R})
$$

with initial distribution

$$
\mu^{n}(0, \mathrm{~d} x)=\frac{1}{n} \sum_{i=1}^{n} \delta_{x_{i}(0)}(\mathrm{d} x) \in M_{1}(\mathbb{R}) .
$$

Remark. The measures $(3.1)-(3.2)$ are concentrated on $(0, \infty) \subseteq \mathbb{R}$. We shall write $M_{1}((0, \infty)), M_{1}([0, \infty))$, $M_{1}(\mathbb{R})$ when we want to emphasize the set where the probability measure under consideration is concentrated.

Definition 3.1. The average of the positions $x_{i}$ of the particles will be denoted by $\bar{x}$. In the special case when $\zeta_{i}(t, \mathbf{x})=p(t, \bar{x})$ for all particles $1 \leq i \leq n$, where $p(t, x)$ is a continuous function $p:[0, \infty) \times(0, \infty) \rightarrow[0,1]$, $\{\mathbf{x}(t)\}_{t \geq 0}$ shall be called a mean field $\gamma$-process.

Throughout this section we assume that $p(t, x)$ is differentiable with bounded derivatives in both variables, $a=1$ and $\lambda=1$ and there exist constants $p_{-}, p_{+}$such that

$$
0<p_{-} \leq p(t, x) \leq p_{+}<1
$$

We now state the general conditions for tightness on the Skorohod space of right-continuous left-limit functions (rcll), which will be needed several times throughout the paper. The parameter $N>0$ eventually converges to infinity; in Sections 3 and $4, N=n$ (the number of particles in the system), while in Section 5 the scaling factor $N$ appears in the space and time coordinates in a nontrivial way.

Let $\left\{Y^{N}(t)\right\}_{t \geq 0}$ be a family of continuous time rcll random process on $\mathbb{R}^{d}$, indexed by $N>0$. For a fixed $N$, the trajectories of the process are elements of the Skorohod space $D\left([0, \infty), \mathbb{R}^{d}\right)$ with the space of continuous paths $C\left([0, \infty), \mathbb{R}^{d}\right)$ as a subset. If

and for any $T>0$ and $\epsilon>0$

$$
\lim _{K \rightarrow \infty} \lim _{N \rightarrow \infty} P\left(\left|Y^{N}(0)\right|>K\right)=0
$$

$$
\lim _{\delta \rightarrow 0} \limsup _{N \rightarrow \infty} P\left(\sup _{0<t-s<\delta, 0 \leq s \leq t \leq T}\left|Y^{N}(t)-Y^{N}(s)\right|>\epsilon\right)=0
$$

then $\left\{Y^{N}(t)\right\}_{t \geq 0}$ is tight in $D\left([0, \infty), \mathbb{R}^{d}\right)$ and any limit point is in $C\left([0, \infty), \mathbb{R}^{d}\right)$. The reader is referred to $[4]$ for the convergence theorems in the Skorohod space.

The fluid limit can be described in closed form by a weak differential-difference equation (3.9) by setting $n=N$ as scaling constant from (3.4)-(3.5). No other quantity, including time $t$, is scaled.

The first assumption (A1) stipulates that the initial mass distribution has a Laplace transform (positive exponential moments). It is trivially satisfied when $\mathbf{x}_{0}$ is deterministic and allows the use of the generalized 
test functions introduced in Definition 2.1. Assumption (A2) is needed to ensure the convergence to an initial mass profile.

Assumption (A1). There exists $\eta_{0}>0$ such that

$$
\limsup _{n \rightarrow \infty} E\left[\left\langle\mathrm{e}^{\eta_{0} x}, \mu^{n}(0, \mathrm{~d} x)\right\rangle\right]<\infty
$$

Assumption (A2). The initial distribution is said to have an initial deterministic profile $\mu_{0}(\mathrm{~d} x) \in M_{1}((0, \infty))$ if $\lim _{n \rightarrow \infty} \mu^{n}(0, \mathrm{~d} x)=\mu_{0}(\mathrm{~d} x)$ in probability in the sense of the weak convergence of finite measures.

Remark. The convergence in probability from (A2) is relevant when the initial configuration is random: in that case, for any $\phi \in C_{b}((0, \infty))$ and $\epsilon>0$,

$$
\lim _{n \rightarrow \infty} P\left(\left|\left\langle\phi, \mu^{n}(0, \mathrm{~d} x)\right\rangle-\left\langle\phi, \mu_{0}(\mathrm{~d} x)\right\rangle\right|>\epsilon\right)=0
$$

Combining (3.7) with (A1) we obtain easily that $\mu_{0}$ necessarily has exponential moments up to $\eta_{0}$ and satisfies the bound (3.6). The next assumption (A3) ensures the de-coupling of individual particles as $n \rightarrow \infty$ stated in Theorem 3.2 .

Assumption (A3). We choose a finite collection of particles $\left\{x_{j}^{n}(\cdot)\right\}, 1 \leq j \leq l$, with $l$ a positive integer fixed for all $n$. Since the limit is considered as $n \rightarrow \infty$ the condition $n \geq l$ is trivial. We assume that for all $1 \leq j \leq l$, the initial point $x_{j}^{n}(0)$ has a deterministic limit $x_{j}$.

In the following, we shall denote $\langle\phi(s), \mu(s)\rangle=\int \phi(s, x) \mu(s, \mathrm{~d} x)$ for a test function $\phi$ and a time-indexed family of measures $\{\mu(t, \mathrm{~d} x)\}_{t \geq 0}$. The test functions defined in Definition 2.1 include the set of $C^{1,2}$ functions continuous up to the origin in the space variable $X$, which allows without any loss of generality to prove tightness in the sense of $(3.4)-(3.5)$ on $[0, \infty)$.

Theorem 3.1. Under (3.3), (A1) and (A2), the average process $\bar{x}^{n}(\cdot)$ is tight in the Skorohod space D([0, $\left.\infty\right)$, $[0, \infty)$ ) and any limit point $\bar{x}(\cdot)$ is the unique deterministic solution of the ordinary differential equation

$$
\frac{\mathrm{d} y}{\mathrm{~d} t}=(1-p(t, y))-(1-\gamma) p(t, y) y, \quad y(0)=\int x \mu_{0}(\mathrm{~d} x) \geq 0
$$

The empirical measure process (3.1) is tight in the Skorohod space of time-indexed measure-valued paths $D([0, \infty)$, $\left.M_{1}([0, \infty))\right)$ and any limit point is the unique solution that verifies the equation

$$
\begin{aligned}
\langle\phi(t), \mu(t)\rangle-\langle\phi(0), \mu(0)\rangle & -\int_{0}^{t}\left\langle\partial_{s} \phi(s, x)+(1-p(s, \bar{x}(s))(\phi(s, x+1)-\phi(s, x))\right. \\
& +p(s, \bar{x}(s))(\phi(s, \gamma x)-\phi(s, x)), \mu(s)\rangle \mathrm{d} s=0,
\end{aligned}
$$

for any $\phi \in C_{\eta}^{1,2}([0, \infty) \times(0, \infty))$.

Remark 3.1. Equation (3.9) is (4.2) in the special case when $A_{t}$ has $p(t, x)=p(t, \bar{x}(t))$. We postpone until Proposition 4.1 in Section 4 to show that the solution in weak sense is unique.

Remark 3.2. Equation (3.8) has unique global solutions (affine ordinary differential equation). Since the right hand side is negative for $y>\frac{\left(1-p_{-}\right)}{(1-\gamma) p_{-}}$, the solution is bounded above. Similarly $y^{\prime}>0$ when $y<\frac{\left(1-p_{+}\right)}{(1-\gamma) p_{+}}$, meaning that the solution is also bounded below away from zero. This shows that the solution $\mu(t, \mathrm{~d} x)$ does not degenerate. 
Remark 3.3. In the case $p(t, x) \equiv p(x)$ (the equation is autonomous) the solution is bounded and monotonic, thus has a limit as $t \rightarrow \infty$. The convergence holds for $p(t, x) \equiv p(t)$ as well when $\lim _{t \rightarrow \infty} p(t)=p$ exists. When $p=\lim _{t \rightarrow \infty} p(t, y(t))$, the particles are approaching a steady state corresponding to the equilibrium distribution of the process. This corresponds to $p$ is constant, a case studied in more detail in the next section.

Remark 3.4. It is rather straightforward to get solutions to certain choices of $p(t, y)$. (i) In the case of time homogeneous $p(t, y)=p(y)$, the equation becomes separable and easy to solve. The equilibrium state $y_{e q}=$ $\lim _{t \rightarrow \infty} y(t)$ can be obtained by solving $y_{e q}=\left(1-p\left(y_{e q}\right)\right) /\left(p\left(y_{e q}\right)(1-\gamma)\right)$. For instance, in case $p(y)=y /(1+y)$, the equilibrium solution is $y_{e q}=(1-\gamma)^{-1 / 2}$ and $p\left(y_{e q}\right)=(1+\sqrt{1-\gamma})^{-1}$. (ii) In the case of state independent $p(t, y)=p(t)$, the explicit solution to the ode is $y(t)=y(0) \mathrm{e}^{-(1-\gamma) \int_{0}^{t} p(u) \mathrm{d} u}+\int_{0}^{t}(1-p(s)) \mathrm{e}^{-(1-\gamma) \int_{s}^{t} p(u) \mathrm{d} u} \mathrm{~d} s$.

Remark 3.5. Similar results to Theorem 3.1 can be obtained for $\zeta_{i}(t, \mathbf{x})=p_{n}(t, \bar{x}, \mathbf{x})$, where $p_{n}(\cdot, \cdot, \cdot)$ approaches a function $p(\cdot, \cdot)$ as in Definition 3.1 with some degree of uniformity in the first two arguments.

Proof. The average process. In this subsection, we apply (3.4)-(3.5) to $N=n, Y^{N}(t)=\bar{x}_{n}(t)$. We recall the coupling between $\mathbf{x}(t)$ and a family of Poisson processes, as in (2.1)-(2.2). We arrange the particles, including the Poisson points, in a set of $n$ pairs $\left(x_{i}(t), \pi_{i}(t)\right), 1 \leq i \leq n$. The Poisson processes are the clocks that trigger the jumps of the particles $x_{i}$, and they only move forward. We then have inequality $x_{i}(t) \leq x_{i}(0)+\pi_{i}(t)$ and (2.1) for all $i$, and

$$
\bar{x}^{n}(t) \leq \bar{x}(0)+\frac{1}{n} \sum_{i=1}^{n} \pi_{i}(t)
$$

The differential equations (2.3)-(2.4) are valid for $f(\mathbf{x}) \in C_{b}\left((0, \infty)^{n}\right)$. Here we are interested in $f(\mathbf{x})=$ $n^{-1} \sum_{i=1}^{n} \phi\left(x_{i}\right)$, with $\phi(x) \in C_{\eta}^{1,2}((0, \infty))$. It is easy to see that we can extend $(2.3)-(2.4)$ to the class of test functions from Definition 2.1, and implicitly to polynomials, due to the exponential bounds (2.1).

Let $T>0$ be fixed but arbitrary and $\phi(x)=x$. At time $t=0$, the average process $\bar{x}_{n}(0)$ is tight (3.6). Then, (2.3)-(2.4) show that for $0 \leq t_{1} \leq t_{2} \leq T$,

$$
E\left[\sup _{t_{1} \leq s \leq t \leq t_{2}}\left|\bar{x}_{n}(t)-\bar{x}_{n}(s)\right|^{2}\right] \leq C\left(t_{2}-t_{1}\right)
$$

with $C=C(T)$ independent of $n$, showing that $\left\{\bar{x}_{n}(\cdot)\right\}_{n \geq 1}$ is tight in $D([0, T],[0, \infty))$, and any limit point $\bar{x}(\cdot)$ is continuous. Moreover, the tight family of processes $\left\{\bar{x}_{n}(\cdot)\right\}_{n \geq 1}$ satisfy, due to Doob's maximal inequality applied to (2.3)-(2.4)

$$
\lim _{n \rightarrow \infty} E\left[\sup _{0 \leq t \leq T}\left|\bar{x}_{n}(t)-\bar{x}_{n}(0)-\int_{0}^{t}\left(1-p\left(s, \bar{x}_{n}(s-)\right)\right)-(1-\gamma) p\left(s, \bar{x}_{n}(s-)\right) \bar{x}_{n}(s-) \mathrm{d} s\right|^{2}\right]=0 .
$$

Assumptions (A2) and (A1) imply that $\bar{x}_{n}(0)$ converges in distribution to the deterministic point $y_{0}=\int x \mu_{0}(\mathrm{~d} x)$. Since $p$ is continuous and bounded, and using once more fact that the expected values of the polynomials $\phi(x)$ have uniform bounds over $n$ and $t \leq T$, we have shown that any limit point $\bar{x}(\cdot)$ solves $(3.8)$. We note that (3.8) has unique local solutions since $p$ is smooth, and since it has an affine bound, it also has global solutions [15].

The fluid limit. The proof of (3.9) follows the same steps as the proof for the average process. One has to prove tightness for $\left\{\mu_{n}(\cdot, \mathrm{d} x)\right\}_{n \geq 1}$, which means that, for any test function $\phi \in C_{b}^{2}((0, \infty))$, the processes $Y_{n}(\cdot)=\left\langle\phi, \mu_{n}(\cdot)\right\rangle$ satisfy both (3.4) and (3.5). The first bound is given by (A1), and the second is a consequence of the Doob's maximal inequality applied to the martingale (2.3). Any limit point of the tight family of measure valued processes (indexed by $n$ ) satisfies equation (3.9) modulo an error term of order $1 / n$. To close the argument, we only need the uniqueness of the solution to the pde (4.2), proven in Proposition 4.1. The details of the proof are standard in any hydrodynamic limit [16], also in a more similar context in [11]. In addition, the proof of Theorem 5.1 in the present paper outlines the main steps of essentially the same argument. 
Theorem 3.2. Under assumptions (A1), (A2), (A3), each particle $\left\{x_{j}^{n}(\cdot)\right\}_{n \geq 1}$ is tight and its limit point is equal in law to the one particle $\gamma$-process defined by equation (2.3) with $n=1$ and $\zeta(t, x)=p(t, \bar{x}(t))$, independent of the space variable $x$, where $\bar{x}(\cdot)$ is the solution of (3.8). Moreover, the joint system of l tagged particles converges to a collection of independent one particle processes starting at $x_{j}, 1 \leq j \leq l$.

Proof. Under (A1), (A2), the average process $\bar{x}_{n}(\cdot)$ converges to the solution of the ordinary differential equation (3.8). It is easy to see that under (A3), that takes care of the initial point, each individual particle is tight. In the Skorohod space $D\left([0, T],(0, \infty)^{l}\right)$, consider the joint $l$-dimensional limit point $\left\{x_{j}(\cdot)\right\}_{1 \leq j \leq l}$ of the tagged particle collection $x_{j}^{n}(\cdot), 1 \leq j \leq l$, obtained as $n \rightarrow \infty$. Taking into account the continuity of the coefficients $p(s, \cdot)$ (to prove that $p\left(s, \bar{x}_{n}(s)\right) \rightarrow p(s, \bar{x}(s))$ ), we see that the $l$-dimensional process $\left\{x_{j}(\cdot)\right\}_{1 \leq j \leq l}$ solves the martingale problem (2.3)-(2.4) with $n=l$, indices $i \rightarrow j, 1 \leq j \leq l$, identical $\zeta_{j}(s, \mathbf{x})=p(t, \bar{x}(t))$ and $\mathbf{x}(0)=\left(x_{1}, x_{2}, \ldots, x_{l}\right)$ (see [12] for details in a similar derivation). Naturally, in this setting the $l$ components are independent since no coefficient of the infinitesimal generator depends on more than one component.

\section{THE ONE PARTICLE PROCESS}

As in Section 3, $a=1$ and $\lambda=1$ and condition (3.3) are in force. We want to investigate the dynamics of the process governed by $(2.3)-(2.4)$ in the special case $n=1$. In view of Definition 3.1, for consistency with Section 3, we denote the jump probabilities $\zeta(t, x)=p(t, x)$. Denote by $A_{t}$ the operator

$$
A_{t} \phi(t, x)=(1-p(t, x))(\phi(t, x+1)-\phi(t, x))+p(t, x)(\phi(t, \gamma x)-\phi(t, x)),
$$

applied to $\phi \in C_{\eta}^{1,2}([0, \infty) \times(0, \infty))$. For a probability measure $\mu_{0}(\mathrm{~d} x)$ on $(0, \infty)$, we say that the time indexed family of measures $\mu(t, \mathrm{~d} x)$ is a weak solution to the evolution equation $\mu_{t}=A_{t}^{*} \mu$ with initial condition $\mu(0, x)=\mu_{0}(\mathrm{~d} x)$, where the star indicates the formal adjoint of $A_{t}$ in the space variable, if

$$
\langle\phi(t, x), \mu(t, \mathrm{~d} x)\rangle-\left\langle\phi(0, x), \mu_{0}(\mathrm{~d} x)\right\rangle=\int_{0}^{t}\left\langle\partial_{s} \phi(s, x)+A_{s} \phi(s, x), \mu(s, \mathrm{~d} x)\right\rangle \mathrm{d} s .
$$

\subsection{The forward equation}

The next proposition looks at the situation when $p(t, x)$ depends only on time. We write $p(t, x)=p(t)$ for simplicity. On one hand, this is a special case of the one particle process, and Proposition 4.1 applies in particular to the case (Thm. 4.1) when the jump rates $\zeta_{i}$ are constant. On the other hand, the interest for this setting comes from the fluid limit in Section 3. By a law of large numbers effect, the average $\bar{x}(t)$ approaches a deterministic limit, as in (3.8). Then, $p(t, \bar{x}(t)) \equiv p(t)$ becomes a function of $t$ only. Uniqueness of the pde (4.2) is essential for closing the argument of the fluid limit from Theorem 3.1.

Proposition 4.1. The solution in weak sense to equation (4.2) exists and is unique and corresponds to the forward equation of the one particle $\gamma$-process with jump rates $\zeta(t, x)=p(t)$ depending continuously of time.

Proof. Existence. The transition probability $\mu(t, \mathrm{~d} x)=P^{\mu_{0}}(x(t) \in \mathrm{d} x)$ of the (nonexplosive) process defined by (2.3)-(2.4) for $n=1$ satisfies the desired equation by taking the expected value in (2.3), which is exactly the forward equation of this particular case of the $\gamma$-process.

Uniqueness. The forward equation in integral form reads

$$
\begin{aligned}
\langle\phi(t, x), \mu(t)\rangle-\langle\phi(0, x), \mu(0)\rangle= & \int_{0}^{t}\left\{\left\langle\partial_{s} \phi(s, x)+(1-p(s))(\phi(s, x+1)-\phi(s, x))\right.\right. \\
& +p(s)(\phi(s, \gamma x)-\phi(s, x)), \mu(s)\rangle\} \mathrm{d} s,
\end{aligned}
$$

and is valid for test functions $\phi(t, x) \in C_{\eta}^{1,2}([0, \infty) \times(0, \infty))$. The bound (2.1) shows that $\eta$ can be arbitrarily large. All we need is that the moment generating functions of all $\mu(t)$ exist on an interval including the origin. 
Next, let $\phi(t, x)=x^{m}$ for integers $m \geq 0$. We can see that $\left\{\left\langle x^{m}, \mu(t)\right\rangle\right\}_{m \geq 0}$ are defined recursively by a system of affine ode's (2.12); such equations have global existence and uniqueness of solutions (see [15]). The induction step uses essentially that $p$ depends on time only. Two solutions will have equal moments for any fixed $t$, so will have equal moment generating functions, thus the measures are the same.

\subsection{The case of constant jump probabilities}

The next theorem gives the exact invariant measure when $\zeta \equiv p(t)=p$ is constant. The particles are trivially independent and it is sufficient to study one individual label $i$, thus $n=1$.

Theorem 4.1. In the case when the jump probabilities are constant with $\zeta(t, x)=p>0$ for all $(t, x)$, the invariant measure $\mu(\mathrm{d} x)$ is unique and has characteristic function

$$
\hat{\mu}(\xi)=E_{\mu}\left[\mathrm{e}^{i \xi x}\right]=\Pi_{n=0}^{\infty}\left(1-\frac{1-p}{p}\left(\mathrm{e}^{i \gamma^{n} \xi}-1\right)\right)^{-1}, \quad \xi \in \mathbb{R} .
$$

Alternatively, let $\left\{W_{k}\right\}_{k \geq 0}$ be i.i.d. geometric random variables with parameter $p$, that is $P\left(W_{n}=j\right)=(1-p)^{j} p$, $j \geq 0$. Then, the probability measure $\mu(\mathrm{d} x)$ on $(0, \infty)$ defined in (4.4) is the distribution of the random variable

$$
X=\sum_{k=0}^{\infty} \gamma^{k} W_{k}
$$

Remark. The form of the invariant measure is essentially the same as in Theorem 2.3.18 (yet the "noise" does not have mean zero) and (4.5) is in the form of a random geometric series as in 2.3.3, both in [7]. Theorem 4.1 is a continuous time result and cannot be directly derived from Theorem 2.3 .18 even after shifting the process by a constant to compensate for the mean value of the noise. It can be used to obtain the invariant measure for the chain given by the position right before each jump.

Proof. The existence and uniqueness of the invariant measure $\mu(\mathrm{d} x)$ is proven in Theorem 2.1 and Proposition 4.1. The functions $x \rightarrow \exp (i \xi x)$ are bounded and continuous; the passage from real-valued to complexvalued functions is elementary. The series (4.5) is convergent in distribution (also almost surely) as long as $0 \leq \gamma<1$, so the distribution of $X$ is well defined. We also notice that the infinite product in (4.4) is convergent due to the inequality $\left|\mathrm{e}^{i \xi}-1\right| \leq 2\left|\sin \frac{\xi}{2}\right| \leq|\xi|$. The invariant measure has to satisfy $\langle A \phi, \mu\rangle=0$ for $A$ the operator (4.1), which is independent of $t$ in this case. Naturally, this is the operator from $(2.3)$ when $n=1$, $\lambda=1, a=1$ and $\zeta=p$ is constant. For $\phi(x)=\exp (i \xi x)$, we verify that the characteristic function $\hat{\mu}(\xi)$ has to satisfy the equation

$$
\left(1-\left(\frac{1-p}{p}\right)\left(\mathrm{e}^{i \xi}-1\right)\right) \hat{\mu}(\xi)=\hat{\mu}(\gamma \xi), \quad \hat{\mu}(0)=1 .
$$

The infinite product from (4.4) is the only solution of this recursive equation.

\section{SCALing Limit}

In this section, we recall the definition of the $\gamma$-process (2.3)-(2.4) with nontrivial choices of intensity $\lambda$ and jump size $a$. Let $N$ be a positive integer. We consider the mean field $\gamma$-process with scaling given by $n=N$, a time speed up $t \rightarrow N t$ given by $\lambda=N$, and the shrinking of the forward jump size $a=N^{-1}$. In addition, we shall assume $p^{N}(t, x)=N^{-1} \alpha(t, x)$, with $\alpha \in C_{b}^{0,1}([0, \infty) \times(0, \infty))$, the space of functions with bounded continuous derivatives up to the multi-index $(0,1)$. Finally, the backward jump size has no scaling on $\gamma \in(0,1)$. The scaled process considered is $\mathbf{x}^{N}(t)=\mathbf{x}(N t)$. Denote the empirical measure by

$$
\mu^{N}(t, \mathrm{~d} x)=N^{-1} \sum \delta_{x_{i}(N t)}(\mathrm{d} x) \in M_{1}((0, \infty))
$$


We recall that for $\phi(t, x) \in C_{\eta}^{1,2}([0, \infty) \times(0, \infty))$ we use the shorthand $\langle\phi(t), \mu(t)\rangle$ for the integral of $\phi$ against the measure $\mu$ over the variable $x$ and that $M_{1}((0, \infty))$ is the space of probability measures on $(0, \infty)$ with the topology of convergence in distribution.

\subsection{Initial profile}

We shall assume that there exists $\eta_{0}>0$, such that

$$
\limsup _{N \rightarrow \infty} E\left[\left\langle\mathrm{e}^{\eta_{0} x}, \mu^{N}(0, \mathrm{~d} x)\right\rangle\right]<+\infty .
$$

Assume that there exists a deterministic measure $\mu_{0}(\mathrm{~d} x) \in M_{1}((0, \infty))$ such that, for any $\epsilon>0$ and any $\phi \in C_{0}^{\infty}((0, \infty))$ we have the limit

$$
\lim _{N \rightarrow \infty} P\left(\left|\left\langle\phi(x), \mu^{N}(0, \mathrm{~d} x)\right\rangle-\left\langle\phi(x), \mu_{0}(\mathrm{~d} x)\right\rangle\right|>\epsilon\right)=0 .
$$

The measure $\mu_{0}(\mathrm{~d} x)$ is called the initial profile of the particle system. Using (5.2) and (5.3) we can show immediately that $\mu_{0}$ must satisfy the same bounds (5.2) on exponential moments up to $\eta_{0}$ as $\mu^{N}(0, \mathrm{~d} x)$. This proves that $\bar{x}^{N}(0)=\left\langle x, \mu^{N}(0, \mathrm{~d} x)\right\rangle$. Even though $\phi(x)=x$ is unbounded, by truncation with a smooth function and monotone convergence, we obtain $\lim _{N \rightarrow \infty}\left\langle x, \mu^{N}(0, \mathrm{~d} x)\right\rangle=\left\langle x, \mu_{0}(\mathrm{~d} x)\right\rangle$ which implies that $z_{0}=\int x \mu_{0}(\mathrm{~d} x)$.

\subsection{The equation satisfied by the average}

For a given $\alpha(t, x), z_{0} \geq 0$, let $z(t)$ be the solution to the ode

$$
z^{\prime}(t)=1-(1-\gamma) \alpha(t, z(t)) z(t), \quad z(0)=z_{0} .
$$

Remark. The solution has global existence and is unique due to the fact that $1-(1-\gamma) \alpha(t, z) z$ is $C^{0,1}$ in $(t, z)$ and affine in $z$. See also the discussion from Remark 3 after Theorem 3.1. As an illustration, let $\alpha$ be constant. In this case $z(t)=r^{-1}\left[1-\left(1-r z_{0}\right) \exp (-r t)\right]$, where $r=\alpha(1-\gamma)>0$, remains bounded for all $t$ and $\lim _{t \rightarrow \infty} z(t)=r^{-1}$.

\subsection{The equation satisfied by the macroscopic profile}

Starting with the solution $z(t)$ of equation (5.4), for a given $\eta>0$ we define the operator $B_{t}$ on the space of test functions $\phi \in C_{\eta}^{1,2}([0, \infty) \times(0, \infty))$

$$
B_{t} \phi(t, x)=\nabla \phi(t, x)+\alpha(t, z(t))(\phi(t, \gamma x)-\phi(t, x)) .
$$

For any $t \geq 0, B_{t}^{*}$ denotes the formal adjoint of $B_{t}$ in the space variable. We shall say that time indexed measures $\mu(t, \mathrm{~d} x)$ satisfy the equation

$$
\partial_{t} \mu=B_{t}^{*} \mu, \quad \mu(0, \mathrm{~d} x)=\mu_{0}(\mathrm{~d} x)
$$

with initial condition $\mu_{0}(\mathrm{~d} x)$ in weak sense, if $\mu(0, \mathrm{~d} x)=\mu_{0}(\mathrm{~d} x)$, and there exists $\eta>0$ such that for any test function $\phi \in C_{\eta}^{1,2}([0, \infty) \times(0, \infty))$,

$$
\langle\phi(t), \mu(t)\rangle-\langle\phi(0), \mu(0)\rangle-\int_{0}^{t}\left\langle\partial_{s} \phi(s)+B_{s} \phi(s), \mu(s)\right\rangle \mathrm{d} s=0 .
$$

A solution $\mu(t, \mathrm{~d} x)$ to (5.7) can be obtained probabilistically as the transition probability of the inhomogeneous Markov process $x(t), t \geq 0$ solving the martingale problem associated to (5.5). The construction can be done either from a scaled pure jump $\gamma$-process, or directly as follows. Let $\chi_{n}, n=1,2, \ldots$ be i.i.d. exponential r.v. 
of intensity one, independent of the starting point $x_{0}>0$. A particle starts at $x_{0}$ and moves at constant speed in the positive direction of the real line. Define $\tau_{1}^{\prime} \leq \infty$ the first time $t>0$ when $\int_{0}^{t} \alpha(s, x(s-)) \mathrm{d} s$ exceeds $\chi_{1}$. If $\tau_{1}^{\prime}=\infty$, the particle moves for all time deterministically at speed one. If $\tau_{1}^{\prime}<\infty, x\left(\tau_{1}^{\prime}\right)=\gamma x\left(\tau_{1}^{\prime}-\right)$. Re-set $x_{0}=x\left(\tau_{1}^{\prime}\right)$ and continue the construction in the same fashion for $\tau_{n}^{\prime}, n \geq 2$. Due to the boundedness of $\alpha$, the process does not end in finite time and the i.i.d. exponentials guarantee that $x(t)$ is Markovian. Once we have constructed the solution $\mu(t, \mathrm{~d} x)$, there is only a question about uniqueness, which is shown in Step 4 of the proof of Theorem 5.1.

Theorem 5.1. Assume both (5.2) and (5.3) and choose $\eta \in\left(0, \eta_{0}\right)$. Then, the average $\bar{x}^{N}(\cdot)$ converges in probability to a deterministic continuous function $z(t)$ solving the equation (5.4). In addition, the measure-valued processes $\mu^{N}(\cdot, \mathrm{d} x)$ converge weakly in probability to the unique measure-valued path $\mu(\cdot, \mathrm{d} x) \in C\left([0, \infty), M_{1}([0, \infty))\right)$ solving the equation (5.6) in weak sense over the space of test functions $C_{\eta}^{1,2}([0, \infty) \times(0, \infty))$.

Proof. We shall prove the theorem on any time interval $[0, T]$, where $T$ is fixed but arbitrary.

Step 1. We want to show that the bounds (5.2) hold for $\mu^{N}(t, \mathrm{~d} x)$, for any $T>0$. First,

$$
\limsup _{N \rightarrow \infty} E\left[\sup _{0 \leq s \leq T}\left\langle\mathrm{e}^{\eta_{0} x}, \mu^{N}(s, \mathrm{~d} x)\right\rangle\right]<+\infty
$$

and then, a fortiori, for all positive integer $m$,

$$
\limsup _{N \rightarrow \infty} E\left[\sup _{0 \leq s \leq T}\left|\left\langle x^{m}, \mu^{N}(s, \mathrm{~d} x)\right\rangle\right|\right]<\infty .
$$

This is based on the martingale inequality applied to the process $\mathbf{x}^{N}(0)+\pi^{N}(t)$ coupled with $\mathbf{x}(t)$. At time $t=0$, both processes start from the same points $x_{i}(0)$. Whenever the exponential clock of intensity $N$ associated to particle $i$ rings, the particle $\pi_{i}$ simply jumps forward by $N^{-1}$. Since $\pi_{i}^{N}(t)$ are independent Poisson processes, all bounds are finite for any $t \leq T$. The uniform integrability from (5.9) follows from (5.8) due to the independence of $\pi^{N}$ and the initial point $\mathbf{x}^{N}(0)$. The bound (5.9) implies that, for any $t \geq 0,\left\{\mu^{N}(t, \mathrm{~d} x)\right\}_{N>0}$ is a tight family of measures.

Step 2. We prove the limit for the average process. First, assume $\phi(x)=x$. Denote $\left\langle\phi, \mu^{N}(t)\right\rangle=z^{N}(t)$ in this case. The bounds (5.8)-(5.9) are stronger than (3.4). Moreover, they allow us to apply the differential formulas (2.3)-(2.4) to $\phi(x)=x$, a polynomial. As a consequence of the martingale maximal inequality, since the quadratic terms (2.4) are of order $N^{-1}$, we obtain (3.5) for $z^{N}(t)$.

Let $z(\cdot)$ be a limit point of $z^{N}(\cdot)$. We note that $z_{0}=\lim _{N \rightarrow \infty}\left\langle x, \mu^{N}(0)\right\rangle=\left\langle x, \mu_{0}\right\rangle$ exists and is unique from (5.3) and an application of (5.2). For $\omega \in D([0, \infty),(0, \infty))$, we write $X_{t}(\omega)=\omega(t)-\omega(0)-\int_{0}^{t}(1-$ $(1-\gamma) \alpha(s, \omega(s-)) \omega(s-) \mathrm{d} s$. Let $X_{t, M}=\left|X_{t}\right| \psi_{M}\left(\left|X_{t}\right|\right)$ where $\psi_{M}$ is a smooth nonnegative version of the indicator function of $[-M, M]$. Here $M>0$ is fixed but arbitrary. The functional $X_{t}$ on $D([0, \infty),(0, \infty))$ is continuous and $X_{t, M}$ is continuous and bounded. We have $\lim _{N \rightarrow \infty} E\left[X_{t, M}\left(z^{N}\right)\right]=E\left[X_{t, M}(z)\right]$. Since $X_{t, M}\left(z^{N}\right) \leq\left|X_{t}\left(z^{N}\right)\right|$ and $\lim _{N \rightarrow \infty} E\left[X_{t}^{2}\left(z^{N}\right)\right]=0$ by Doob's maximal martingale inequality, we conclude that $E\left[\left|X_{t}(z)\right|\right]=0$ after $M \rightarrow \infty$. Finally $X_{t}(z)=0$ with probability one. The random process $X_{t}(z), t \geq 0$ is also continuous with probability one. With the exception of a set of probability zero, $X$. $(z)$ is continuous on $[0, T]$ and for all $t \in \mathbb{Q} \cap[0, T], X_{t}(z)=0$. This implies that $\sup _{t \in[0, T]}\left|X_{t}(z)\right|=0$ with probability one. We want to prove a little more:

$$
\lim _{N \rightarrow \infty} E\left[\sup _{s \in[0, T]}\left|\bar{x}^{N}(s)-z(s)\right|\right]=0 .
$$

The functional $\omega(\cdot) \rightarrow F(\omega)=\sup _{s \in[0, T]}\left|\omega(s)-g_{0}(s)\right|$ is continuous on $D([0, T], \mathbb{R})$ when $g_{0}(\cdot)$ is continuous - from [4]. In this case, set $z(s)=g_{0}(s)$ and $\omega(s)=\bar{x}^{N}(s)$. This implies that $F_{M}(\omega)=F(\omega) \psi_{M}(F(\omega))$ is continuous and bounded. The convergence in distribution, followed by $M \rightarrow \infty$ concludes the proof. 
Step 3. Let $\phi \in C_{\eta}^{1,2}([0, \infty) \times(0, \infty))$. For each $N$, the differential formula corresponding to $\left\langle\phi(t), \mu^{N}(t)\right\rangle$ can be obtained directly from (2.3), applied to the function $f(t, \mathbf{x})=N^{-1} \sum \phi\left(t, x_{i}\right)$. Formulas (2.3)-(2.4) give (recall $n=N$ and $p^{N}(s, x)=N^{-1} \alpha(s, x), \alpha$ continuous in $s$ )

$$
\begin{array}{r}
\mathcal{M}_{\phi}^{N}(t)=\left\langle\phi(t), \mu^{N}(t)\right\rangle-\left\langle\phi(0), \mu^{N}(0)\right\rangle \\
-\int_{0}^{t} N^{-1} \sum_{i=1}^{N}\left\{\partial_{s} \phi\left(s, x_{i}^{N}(s-)\right)\right. \\
+N\left[\left(1-p^{N}\left(s, \bar{x}^{N}(s-)\right)\right)\left(\phi\left(s, x_{i}^{N}(s-)+\frac{1}{N}\right)-\phi\left(s, x_{i}^{N}(s-)\right)\right)\right. \\
\left.\left.+p^{N}\left(s, \bar{x}^{N}(s-)\right)\left(\phi\left(s, \gamma x_{i}^{N}(s-)\right)-\phi\left(s, x_{i}^{N}(s-)\right)\right)\right]\right\} d s .
\end{array}
$$

(1) The integrands in (5.12)-(5.14) have uniformly bounded moments due to the estimates (5.8). This proves that $\left\langle\phi(\cdot), \mu^{N}(t)\right\rangle$, indexed by $N$, satisfy (3.4)-(3.5), showing that $\left\{\mu^{N}\right\}_{N>0}$ are tight processes on $D\left([0, T], M_{1}([0, \infty))\right.$, and any limit point belongs to $C\left([0, T], M_{1}([0, \infty))\right)$.

(2) Modulo error terms of order $N^{-1}$, line (5.13) is equal to $\nabla \phi\left(x_{i}^{N}(s)\right)$. Here we use that $\alpha \in C_{b}^{0,1}([0, \infty) \times$ $(0, \infty)$ ), and then Taylor's formula with the remainder in integral form.

(3) Given $\phi(t, x)$, denote $\phi_{\gamma}(s, x)=\phi(s, \gamma x)$. For $\nu \in D\left([0, T], M_{1}([0, \infty))\right.$, define

$$
\begin{gathered}
U_{\phi}(s)=\left\langle\partial_{s} \phi(s)+\nabla \phi(s)+\alpha(s, z(s))\left(\phi_{\gamma}(s)-\phi(s)\right), \nu(s-)\right\rangle ; \\
X(\nu)=\langle\phi(t), \nu(t)\rangle-\langle\phi(0), \nu(0)\rangle-\int_{0}^{t} U_{\phi}(s) \mathrm{d} s .
\end{gathered}
$$

We would like to prove that $\lim _{N \rightarrow \infty} E\left[\left|X\left(\mu^{N}\right)\right|\right]=0$. By Doob's maximal inequality, we have $\lim _{N \rightarrow \infty}$ $E\left[\left|X^{-}\left(\mu^{N}\right)\right|^{2}\right]=0$, where $X^{-}$is the functional identical to $X$ except the factor $\alpha(s, z(s))$ in $U_{\phi}(s)$ that is replaced by $\alpha\left(s, \bar{x}^{N}(s-)\right)$. To evaluate $E\left[\left|X^{-}\left(\mu^{N}\right)-X\left(\mu^{N}\right)\right|\right]$, we shall apply Hölder's inequality inside the time integral containing $U_{\phi}$. Due to the fact that $\alpha(t, x)$ is bounded and $\phi(s, x)$ has exponential moments for some $\eta_{0}>0$, it is sufficient to show that, for a suitable $r>1$,

$$
\lim _{N \rightarrow \infty} E\left[\sup _{s \in[0, T]}\left|\alpha\left(s, \bar{x}^{N}(s-)\right)-\alpha(s, z(s))\right|^{r}\right]=0 .
$$

For any $r>1$, the limit (5.16) results from (5.10) and dominated convergence. For the exponential moments (5.2) we need $\eta \leq \eta_{0}(1-1 / r)$, which shows that the only condition on $\eta$ is $\eta<\eta_{0}$.

From here on we proceed like in Step 2, first by truncation with $\psi_{M}(x)$, a smooth version of the indicator function of $[-M, M]$. For ease of notation, we omit the subscript $t$ until the end of this paragraph. The functional $X_{M}(\nu)=|X(\nu)| \psi(X(\nu))$, defined in similar fashion to $X_{t, M}$ in Step 2, is continuous and bounded. Since $\lim _{N \rightarrow \infty} E\left[\left|X\left(\mu^{N}\right)\right|^{2}\right]=0$, we derive that $E[|X(\mu)|]=0$ for any limit point $\mu \in C\left([0, \infty) \times M_{1}([0, \infty))\right.$ ), following the reasoning from Step 2. So $X(\mu)=0$ with probability one. At this point we recall the dependence on $t$ of the random variable $X(\nu)=X_{t}(\nu)$. By continuity, exactly as at Step 2, we conclude that $\sup _{t \in[0, T]}|X(\mu)|=0$ with probability one.

(4) We know that any limit point $\mu(\cdot, \mathrm{d} x)$ of the tight family of empirical measures $\left\{\mu^{N}(\cdot, \mathrm{d} x)\right\}_{N>0}$ is continuous in time and satisfies equation (5.6) in weak sense. We only have to prove that the equation has a unique deterministic solution, completed in the next step.

Step 4. To prove the uniqueness of the solution to (5.6), for any weak solution of (5.7), we define the exponential moment $\left\langle\exp \left(\eta_{0} x, \mu(t, \mathrm{~d} x)\right\rangle\right.$ and the moments $\mu_{m}(t)=\left\langle x^{m}, \mu(t, \mathrm{~d} x)\right\rangle, m \geq 0$. The moments are finite for a limit point $\mu$ due to (5.8) via the same truncation argument used in Step 2. The existence of a positive exponential moment shows that the moment generating function is defined on an open interval containing the origin. 
Hence the measure $\mu$ is identified by its moments. The (5.7) applied to $\phi(t, x)=x^{m}$ gives the recurrence

$$
\frac{\mathrm{d}}{\mathrm{d} t} \mu_{m}(t)=m \mu_{m-1}(t)+\alpha(t, z(t))\left(\gamma^{m}-1\right) \mu_{m}(t), \quad \mu_{0}(t)=1
$$

The affine ode's have unique global solutions since $\alpha$ is bounded (a general result when the equation has an affine bound [15]). For two limit points $\mu^{\prime}$ and $\mu^{\prime \prime}$, the equality of moments implies $\mu^{\prime}=\mu^{\prime \prime}$.

Acknowledgements. We would like to thank D.Y. Eun from North Carolina State University for introducing us to the subject of internet congestion control and the anonymous referee for valuable suggestions on presentation and the reference list.

\section{REFERENCES}

[1] G. Appenzeller, I. Keslassy and N. McKeown, Sizing router buffer. In Proc. of the 2004 Conf. on Applications, Technologies, Architectures, and Protocols for Computers Communications, Portland, OR, USA. ACM New York, NY (2004), pp. 281-292.

[2] F. Baccelli, D. McDonald and J. Reynier, A mean-field model for multiple TCP connections through a buffer implementing RED. Performance Evaluation Archive 49 (2002) 77-97.

[3] F. Baccelli, A. Chaintreau, D. De Vleeschauwer and D. McDonald, A mean-field analysis of short lived interacting TCP flows. In Proc. of the Joint Int. Conf. on Measurement and Modeling of Computer Systems, New York, NY, USA, June 10-14, 2004 (SIGMETRICS '04/Performance '04). ACM New York, NY (2004), pp. 343-354.

[4] P. Billingsley, Convergence of Probability Measures. Wiley Series in Probability and Statistics, New York (1999). Ch. 3, pp. 109-153 or more precisely, Ch. 3.15, pp. 123-136.

[5] H. Cai and D.Y. Eun, Stability of Network Congestion Control with Asynchronous Updates. In Proc. IEEE CDC 2006, San Diego, $C A$ (2006).

[6] A. Dhamdere and C. Dovrolis, Open issues in router-buffer sizing. ACM SIGCOMM Comput. Commun. Rev. 36. ACM New York, NY (2006) 87-92.

[7] M. Duflo, Random iterative models. Volume 34 of Applications of Mathematics (New York). Springer-Verlag, Berlin (1997).

[8] V. Dumas, F. Guilleaumin and P. Robert, A Markovian analysis of Additive-Increase Multiplicative-Decrease (AIMD) algorithms. Adv. Appl. Probab. 34 (2002) 85-111.

[9] D.Y. Eun, On the limitation of fluid-based approach for internet congestion control. In Proc. IEEE Int. Conf. on Computer Communications and Networks, ICCCN, San Diego, CA, USA. J. Telecommun. Syst. 34 (2007) 3-11.

[10] D.Y. Eun, Fluid approximation of a Markov chain for TCP/AQM with many flows. Preprint.

[11] I. Grigorescu and M. Kang, Hydrodynamic Limit for a Fleming-Viot Type System. Stoch. Process. Appl. 110 (2004) 111-143.

[12] I. Grigorescu and M. Kang, Tagged particle limit for a Fleming-Viot type system. Electron. J. Probab. 11 (2006) 311-331 (electronic).

[13] I. Grigorescu and M. Kang, Recurrence and ergodicity for a continuous AIMD model. Preprint.

[14] F. Guillemin, P. Robert and B. Zwart, AIMD algorithms and exponential functionals. Ann. Appl. Probab. 14 (2004) $90-117$.

[15] J.K. Hale, Ordinary Differential Equations. Wiley-Interscience, New York (1969).

[16] C. Kipnis and C. Landim, Scaling Limits of Interacting Particle Systems. Springer-Verlag, New York (1999).

[17] K. Maulik and B. Zwart, An extension of the square root law of TCP. Ann. Oper. Res. 170 (2009) 217-232.

[18] S.P. Meyn and R.L. Tweedie, Markov chains and stochastic stability. Communications and Control Engineering Series. Springer-Verlag, London, Ltd. (1993).

[19] T.J. Ott and J. Swanson, Asymptotic behavior of a generalized TCP congestion avoidance algorithm. J. Appl. Probab. 44 (2007) 618-635. 